\title{
cAMP-Dependent Protein Kinase Phosphorylations on Tau in Alzheimer's Disease
}

\author{
Gregory A. Jicha, ${ }^{1}$ Charles Weaver, ${ }^{2}$ Eric Lane, ${ }^{1}$ Cintia Vianna, ${ }^{1}$ Yvonne Kress, ${ }^{1}$ Julia Rockwood, ${ }^{1}$ \\ and Peter Davies ${ }^{1,2}$ \\ Departments of ${ }^{1}$ Pathology and ${ }^{2}$ Neuroscience, Albert Einstein College of Medicine, Bronx, New York 10461
}

To elucidate the role cAMP-dependent protein kinase (PKA) phosphorylations on tau play in Alzheimer's disease, we have generated highly specific monoclonal antibodies, CP-3 and PG-5, which recognize the PKA-dependent phosphorylations of ser214 and ser409 in tau respectively. The present study demonstrates by immunohistochemical analysis, CP-3 and PG-5 immunoreactivity with neurofibrillary pathology in both early and advanced Alzheimer's disease, but not in normal brain tissue and demonstrates that cAMP-dependent protein kinase phosphorylations on tau precede or are coincident with the initial appearance of filamentous aggregates of tau. Studies

Abnormally hyperphosphorylated tau is the major proteinaceous constituent of the neurofibrillary pathology seen in Alzheimer's Disease (AD) (Grundke-Iqbal et al., 1986; Wood et al., 1986; Lee et al., 1991; Goedert et al., 1992; Kanemaru et al., 1992). Hyperphosphorylation of tau, both in vitro and in vivo, has been shown to decrease the affinity of tau for microtubules leading to disruption of the neuronal cytoskeleton and axonal transport mechanisms (Goedert and Jakes, 1990; Drechsel et al., 1992; Raffaelli et al., 1992; Biernat et al., 1993; for review, see Lee, 1993; Scott et al., 1993; Yoshida and Ihara, 1993; Brandt et al., 1994; Leger et al., 1997; Illenberger et al., 1998). Phosphorylation also inhibits the degradation of tau leading to its gradual accumulation in the cell (Vincent et al., 1994). Certain phosphorylations have also been shown to substantially decrease the electrophoretic mobility of tau by SDS-PAGE analysis over that expected for the accumulation of additional phosphates (presumably the result of conformational alterations in tau) (Scott et al., 1993; Brandt et al., 1994; Leger et al., 1997). Although many neuronal kinases have been implicated as responsible for each of the above functional consequences of tau phosphorylation, the actions of cAMP-dependent protein kinase (PKA) alone can account for all (Scott et al., 1993; Brandt et al., 1994; Leger et al., 1997; Illenberger et al., 1998). As such, a thorough characterization of PKA-dependent phosphorylations on tau is essential for understanding of the pathogenesis of neurofibrillary degeneration seen in $\mathrm{AD}$ and other tau-related neurodegenerative disorders.

\footnotetext{
Received Jan. 26, 1999; revised June 21, 1999; accepted June 22, 1999.
}

This work was supported by National Institute of Mental Health Grant 38623, National Institutes of Health Grant AG06803, National Institutes of Health training Grants T32GM07288, AG00194, and NS07098, and a grant from Molecular Geriatrics Corporation. We thank M. Goedert for providing the htau40 construct, J. Wang for providing activated $\mathrm{Cdk} 5$, and J. Erlichmann for providing the anti-PKA-RII $\beta$ (Ab40) monoclonal antibody.

Correspondence should be addressed to Dr. Peter Davies, Departments of Pathology and Neuroscience, Albert Einstein College of Medicine, 1300 Morris Park Avenue, Forchheimer 526, Bronx, NY 10461.

Copyright (C) 1999 Society for Neuroscience $0270-6474 / 99 / 197486-09 \$ 05.00 / 0$ using heat-stable preparations demonstrate that neither site appears to be phosphorylated to any appreciable extent in normal rodent or human brain. Further analysis demonstrates that the $\beta$ catalytic subunit of $\operatorname{PKA}(\mathrm{C} \beta)$, the $\beta$ II regulatory subunit of PKA (RII $\beta)$, and the $79 \mathrm{kDa} A$-kinase-anchoringprotein (AKAP79), are tightly associated with the neurofibrillary pathology, positioning CAMP-dependent protein kinase to participate directly in the pathological hyperphosphorylation of tau seen in Alzheimer's disease.

Key words: tau; Alzheimer's disease; protein kinase; cAMP; phosphorylation; neurofibrillary pathology
PKA in the unactivated state exists as a heterotetramer of two catalytic subunits bound to two regulatory subunits. After activation of adenylate cyclase via extracellular signaling events, ATP is converted to $\mathrm{CAMP}$ which then binds to the regulatory subunits releasing the now-activated catalytic subunits of PKA which in turn phosphorylate substrate proteins (for review, see Walsh and Van Patten, 1994). Which proteins become phosphorylated by PKA is largely determined by the subcellular localization of the holoenzyme complex, and this is mediated by a family of proteins termed A-kinase-anchoring proteins (AKAPs) which tether the regulatory subunits of PKA to specific intracellular structures (for review, see Coghlan et al., 1993). Thus, effective signal transduction is dependent on both elevation of intracellular cAMP levels and the localization of PKA in a complex with specific substrates.

Two highly specific monoclonal antibodies, CP-3 and PG-5, have been generated that recognize the PKA-dependent phosphorylations of ser214 and ser409 on tau. This study includes a characterization of these reagents and a demonstration that these phosphorylations are associated with neurofibrillary pathology in AD. Neither site appears to be phosphorylated to any appreciable extent in normal adult rodent or adult human brain. Additional data demonstrate that the $\beta$ catalytic subunit of PKA $(\mathrm{C} \beta)$, the $\beta$ II regulatory subunit of PKA (RII $\beta)$, and the $79 \mathrm{kDa}$ A-kinaseanchoring-protein (AKAP79) colocalize with and are tightly bound to paired helical filaments (PHF) from AD brain. These data demonstrate that the PKA holoenzyme is uniquely positioned to participate in the neurofibrillary degeneration characteristic of $\mathrm{AD}$, and that PKA-dependent phosphorylations on tau can be used to monitor the pathological conversion of normal tau into an AD-like state.

\section{MATERIALS AND METHODS}

Human tissues. Brain tissue was obtained at biopsy and autopsy by the Neuropathology Specimen Bank of Albert Einstein College of Medicine. Autopsy tissues were obtained 3-15 hr after death, and fixed in formalin for at least 3 weeks before sectioning. Biopsy brain tissue was apparently 


\begin{tabular}{ll}
\hline \multicolumn{2}{l}{ Table 1. Sequences of phospho-tau and non-phospho-tau peptides } \\
Peptide & Sequence \\
\hline Ser46(P) & Biotin-KTDAGLKES(P)PLQTPTE \\
Ser202(P) & Biotin-KSGYSSPGS(P)PGTPGSR \\
Ser214(P) & Biotin-KGSRSRTPS(P)LPTPPTRE \\
Thr231 & Biotin-KKVAVVRTPPKSPS \\
Thr231(P) & Biotin-KKVAVVRT(P)PPKSPSS \\
Ser235(P) & Biotin-KVAVVRTPPS(P)PSSAKSR \\
Ser262(P) & Biotin-KNVKSKIGS(P)TENLKHQ \\
Ser356(P) & Biotin-KRVQSKIGS(P)LDNITHV \\
Thr361(P) & Biotin-KIGSLDNIT(P)HVPGGGN \\
Ser409 & Biotin-KGDTSPRHLSNVSSTGSID \\
Ser409(P) & Biotin-KGDTSPRHLS(P)NVSSTGSID \\
Ser412(P) & Biotin-KGDTSPRHLSNVS(P)STGSID \\
Ser413(P) & Biotin-KGDTSPRHLSNVSS(P)TGSID \\
Ser416(P) & Biotin-KGDTSPRHLSNVSSTGS(P)ID \\
Ser422(P) & Biotin-KGSIDMVDS(P)PQLATLA \\
\end{tabular}

normal temporal cortex obtained from patients undergoing temporal lobectomy for intractable epilepsy. For this study, hippocampal tissue from 11 cases of Alzheimer's disease and four normal individuals was used. Alzheimer cases were selected from cases ranging from Braak stage 2 to stage 5. One of the normal individuals had neurofibrillary tangles confined to the transentorhinal cortex, and was thus classified as Braak stage 1 . These cases are representative of a very large number that have been examined with CP-3 and PG-5 for other studies. Vibratome sections of hippocampus, frontal cortex, and temporal cortex from a total of 40 individuals have been investigated with these antibodies. Similar staining is obtained with these antibodies on formalin-fixed, paraffin-embedded tissue sections, and samples from over 120 individual cases have been examined.

Recombinant tau and phosphorylation reactions. Clone htau40 was the generous gift of M. Goedert (Goedert et al., 1989). Clone htau 40 was digested with $N d e$ I and EcoRI, blunted with Klenow, and ligated into SmaI-digested pQE-32 (Qiagen, Hilden, Germany) to produce a histidine-tagged recombinant protein, TauW. TauW was chemically transformed into Escherichia coli strain MC-15 harboring the pREP4 plasmid. Recombinant TauW synthesis was induced, and the protein was purified under nondenaturing conditions according to the manufacturer's specifications. The eluted sample was then dialyzed overnight in three changes of TBS in preparation for phosphorylation reactions. Protein concentrations were assayed by the method of Bradford, and concentrations were adjusted to $0.2 \mathrm{mg} / \mathrm{ml}$.

PKA and GSK-3 were purchased from Upstate Biotechnology (Lake Placid, NY), and reactions were carried out according to the supplier's specifications. Recombinant PKC (catalytic subunit) was purchased from Calbiochem (La Jolla, CA). Activated cdk5 was the generous gift of Dr. J. Wang and was reacted as described previously (Paudel et al., 1993). Kinase activity was assessed against recombinant TauW and adjusted to allow for roughly equivalent levels of phosphate incorporation (2.4-3.7 $\mathrm{mol} \mathrm{PO}_{4} / \mathrm{mol} \mathrm{TauW}$ ) with each kinase.

Monoclonal antibodies. CP-3 was generated by immunizing mice with affinity-purified paired helical filaments from AD brain tissue as described previously (Jicha et al., 1999). PG-5 was generated by immunizing mice with the synthetic phosphopeptides KSPRHLS(P)NVS(P)STGS(P)ID and KSPRHLS(P)NVSS(P)TGSID. After immunization, spleens were dissected and fused to NSO cells using PEG 4000 (de St. Groth and Scheideggers, 1980). Hybridomas were screened by solutional ELISA using a series of biotinylated phospho-tau and non-phospho-tau peptides (Table 1) and biotinylated affinity-purified PHF. CP-3, an IgM isotype, was found to react with only PHF and the tau peptide containing phospho-ser214 (Fig. 1). Similar results have been obtained with two other antibodies (CP12 and CP15) with the same specificity. PG-5, an IgG3 isotype, was found to react with only PHF and the tau peptide containing phospho-ser409 (Fig. 1). A total of nine antibodies specifically reactive with the phosphoserine 409 peptide were also obtained. PHF-1 monoclonal antibody, an IgG1 isotype recognizing phospho-ser396 and phospho-ser404 in tau (Greenberg et al., 1992; Otvos et al., 1994), and

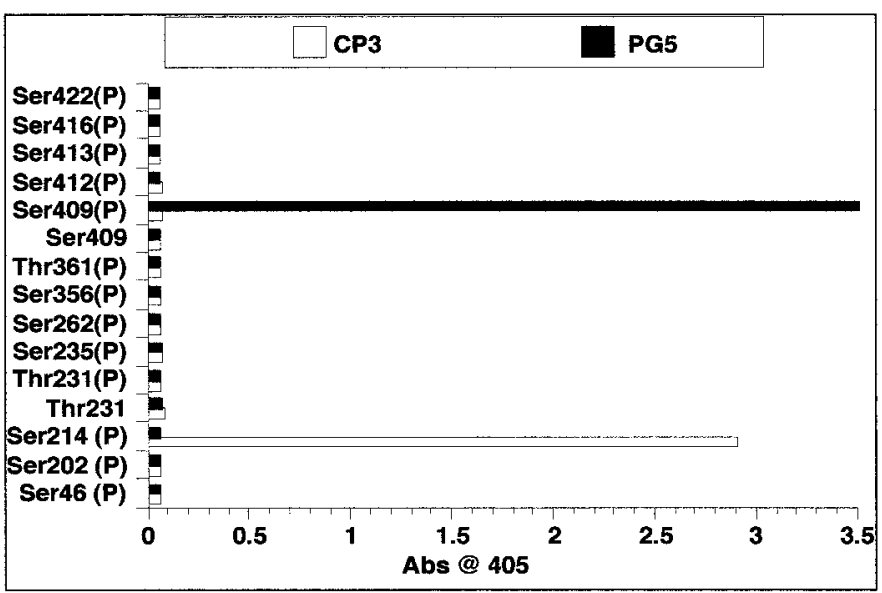

Figure 1. CP-3 and PG-5 monoclonal antibody specificity. ELISA data demonstrating the specificity of CP-3 for phospho-ser214 and PG-5 for phospho-ser409. Peptide sequences and identification of phosphorylated residues are provided in Table 1.

CP-13 monoclonal antibody, an IgG1 isotype recognizing phosphoser202 in tau, were used as positive controls for PHF and phosphorylated tau. TG-5, an IgG1 isotype monoclonal antibody recognizing a phosphorylation-independent primary sequence epitope in tau between amino acids 220-242 was used as a positive control for total tau (Jicha et al., 1997a). CP-3, CP-13, PG-5, PHF-1, and TG-5 were used at a 1:10 dilution. The anti-AKAP79 and anti-calcineurin monoclonal antibodies were purchased from Transduction Laboratories (Lexington, KY) and were used according to manufacturer's specifications. The anti-PKA-C $\beta$ polyclonal antibody was purchased from Santa Cruz Biotechnology (Santa Cruz, CA) and was used according to manufacturer's specifications. The anti-RII $\beta$-specific mouse monoclonal (Ab40) was the generous gift of Dr. J. Erlichmann and was used at 1:1000 for Western blot analyses (Licameli et al., 1992). All secondary antibodies were purchased from Fisher Scientific (Houston, TX) and were used at 1:500 dilution.

Sample preparation and Western blot analyses. Immunoaffinity-purified PHF was prepared as described previously (Jicha et al., 1999). All samples were run on $10 \%$ polyacrylamide gels after solublization in sample buffer and heating at $95^{\circ} \mathrm{C}$ for $5 \mathrm{~min}$. Molecular weights were verified using prestained markers obtained from Life Technologies (Bethesda, MD). Proteins were then transferred to $0.45 \mu \mathrm{M}$ pore nitrocellu-

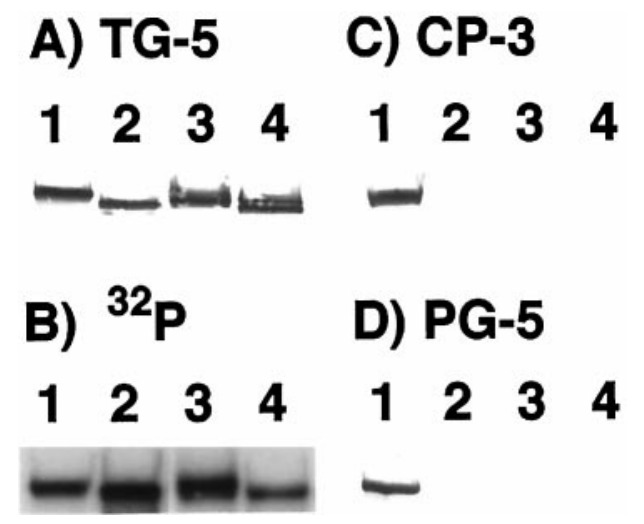

Figure 2. CP-3 and PG-5 recognize PKA-dependent phosphorylations on tau. The CP-3 and PG-5 epitopes are created by PKA (lane 1 ) but not PKC (lane 2), Cdk5 (lane 3), or GSK-3 $\beta$ (lane 4) on recombinant tau. $A$, TG-5 Western blot demonstrating equivalent loading of in vitro phosphorylated recombinant TauW. B, Autoradiogram showing equivalent levels of phosphate incorporation by PKA, PKC, Cdk5, and GSK-3 $\beta$ on recombinant TauW. $C, \mathrm{CP}-3$ Western blot demonstrating epitope creation on recombinant TauW by PKA, but not PKC, Cdk5, or GSK-3 $\beta . D$, PG-5 Western blot demonstrating epitope creation on recombinant TauW by PKA, but not PKC, Cdk5, or GSK-3 $\beta$. 


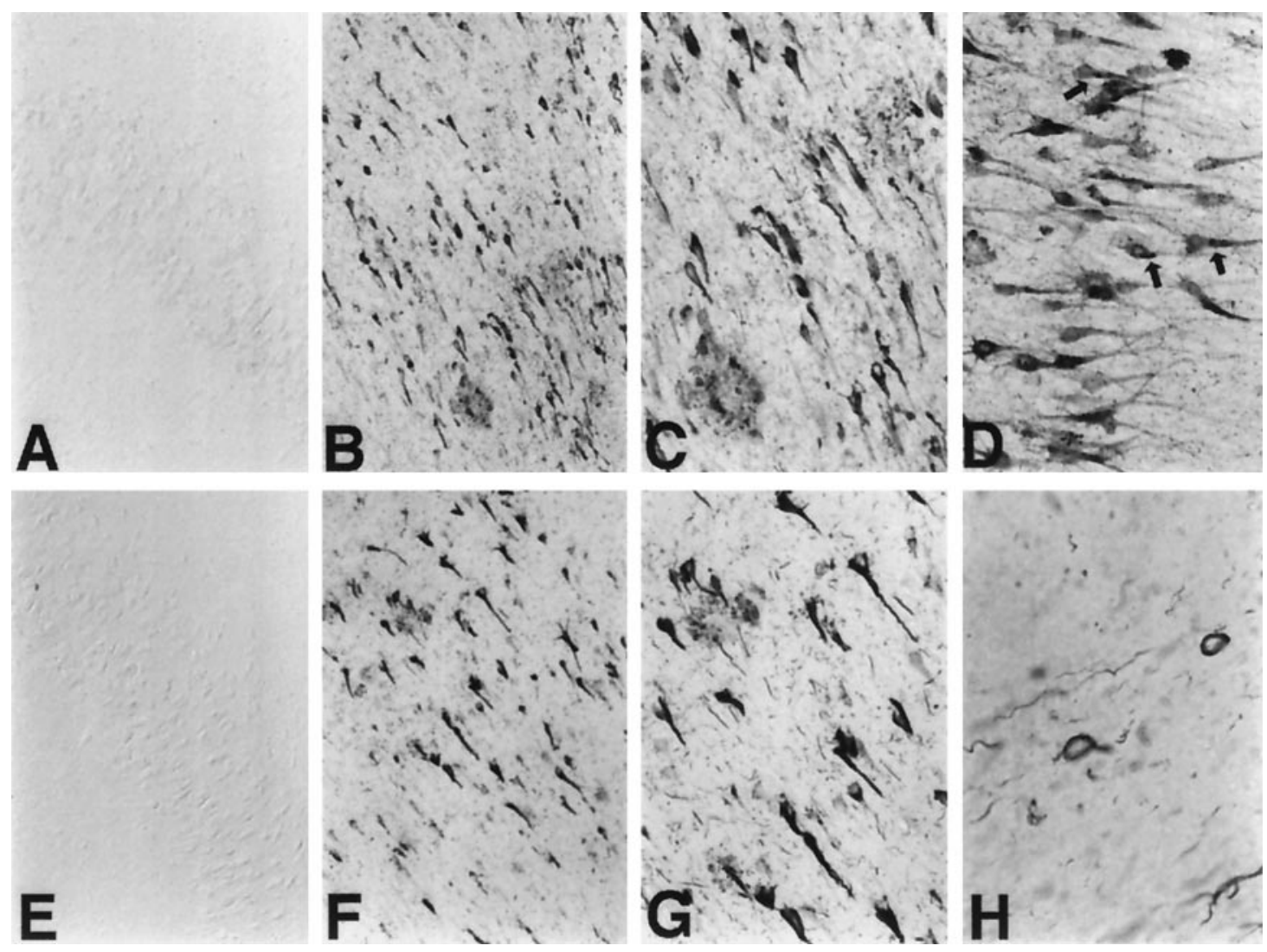

Figure 3. CP-3 and PG-5 react specifically with the neurofibrillary pathology characteristic of AD. A, Normal adult human autopsy tissue is devoid of CP-3 reactivity. $B$, Low-power view $(10 \times)$ of neurofibrillary tangles, dystrophic neurites, and senile plaques in AD brain stained with $C P-3$. $C$, High-power view $(40 \times)$ of $B . D, C P-3$-reactive neurons in $\mathrm{AD}$ hippocampal subfield CA2 exhibit rounded foci of immunoreactivity (arrows) in the cell body near the proximal portion of the apical dendrite. $E$, Normal adult human autopsy tissue is devoid of PG-5 reactivity. $F$, Low-power view (10 $\times$ ) of neurofibrillary tangles, dystrophic neurites, and senile plaques in AD brain stained with PG-5. $G$, High-power view $(40 \times)$ of $F$. $H$, PG-5 reacts with what appear to be "coiled bodies" or oligodendrocytic tau inclusions in the white matter of many AD cases. All panels except $H$ are from CA2 of hippocampus; $H$ is white matter beneath the hippocampus.

lose in preparation for Western blot analysis. Blots were blocked in 5\% milk in TBS for $30 \mathrm{~min}$ and incubated in primary antibody diluted in 5\% milk in TBS for $16 \mathrm{hr}$ at $4^{\circ} \mathrm{C}$. Horseradish peroxidase-conjugated isotype-specific anti-mouse antibodies were used for detection of primary antibody binding and were incubated for $2 \mathrm{hr}$ at $25^{\circ} \mathrm{C}$. Immunostained proteins were visualized by reaction with either 4-chloronapthal (Sigma, St. Louis, MO) $0.5 \mathrm{mg} / \mathrm{ml}$ in the presence of $\mathrm{H}_{2} \mathrm{O}_{2}$ or with the Pierce (Rockford, IL) Super Signal Ultra chemiluminescence detection kit.

ELISA analysis. A series of phospho-tau and non-phospho-tau peptides were synthesized with an N-terminal biotin tag (Table 1). 96-well plates (Nunc, Roskilde, Denmark) were coated with NeutrAvidin (Pierce) for $3 \mathrm{hr}$ at $37^{\circ} \mathrm{C}$. The plates were blocked with $2 \%$ BSA in TBS for $1 \mathrm{hr}$ at $25^{\circ} \mathrm{C}$. Peptides were diluted to $1 \mu \mathrm{M}$ in $2 \%$ BSA in TBS and $50 \mu \mathrm{l}$ per well was incubated for $1 \mathrm{hr}$ at $25^{\circ} \mathrm{C}$. Plates were washed with $0.1 \%$ Tween 20 in TBS and incubated with CP-3 or PG-5 for $2 \mathrm{hr}$ at $25^{\circ} \mathrm{C}$ at a 1:50 dilution in 2\% BSA in TBS. Plates were again washed and incubated for $2 \mathrm{hr}$ at $25^{\circ} \mathrm{C}$ with HRP-labeled goat anti-mouse isotypespecific secondary antibodies at a 1:500 dilution in 2\% BSA in TBS. Plates were washed and developed with Bio-Rad (Hercules, CA) ABTS peroxidase substrate. Optical density was measured at $405 \mathrm{~nm}$ using an SLT Spectra plate reader (Tecan Technicals US).

Immunohistochemistry. Vibratome sections of formalin-fixed $\mathrm{AD}$ and normal hippocampus $(50-\mu \mathrm{m}$-thick) were incubated in $3 \%(\mathrm{vol} / \mathrm{vol})$ hydrogen peroxide $/ 0.25 \%$ Triton $\mathrm{X}-100$ for $30 \mathrm{~min}$ at room temperature and washed in $10 \mathrm{~mm}$ Tris and $150 \mathrm{~mm} \mathrm{NaCl}, \mathrm{pH} 7.4$ (TBS). Sections were then incubated in 5\% nonfat dry milk (wt/vol) in TBS (TBS-milk) for $1 \mathrm{hr}$ at room temperature to block nonspecific antibody binding and incubated in dilutions of either CP-3, PG-5, or anti-AKAP79 in TBSmilk for $16 \mathrm{hr}$ at $4^{\circ} \mathrm{C}$. Tissue sections were then washed with several changes of TBS and reincubated with HRP-labeled isotype-specific secondary antibodies at a 1:500 dilution in TBS-milk for $2 \mathrm{hr}$ at room temperature. Tissue sections that were double-labeled were also incubated with an alkaline phosphatase-conjugated secondary antibody at 1:500 dilution in TBS-milk for $2 \mathrm{hr}$ at room temperature. Tissue sections were then washed with several changes of TBS, and antibody-binding of peroxidase-labeled secondaries was visualized by reaction with $0.3 \mathrm{mg} / \mathrm{ml}$ diaminobenzidine (Sigma) in the presence of $\mathrm{H}_{2} \mathrm{O}_{2}$. Double-labeled sections were also incubated with alkaline phosphatase-labeled secondaries and reacted with NBT/BCIP (Pierce). Tissue sections were then mounted on gelatin-coated slides, dehydrated in ethanol and xylene, and coverslipped.

Electron microscopy. Immunostained vibratome tissue sections of early AD hippocampus were microdissected, post-fixed in $1 \% \mathrm{OsO}_{4}$, dehydrated in ascending ethanol solutions, and embedded in Epon Araldite. Thin sections were prepared and examined unstained using a JEOL Jem 100CX electron microscope.

\section{RESULTS}

To determine the specificity of both CP-3 and PG-5 for phosphoser214 and phospho-ser409, respectively, antibody reactivity toward a panel of synthetic phospho-tau and non-phospho-tau peptides (Table 1) was assessed by ELISA (Fig. 1). CP-3 was shown to react with only the peptide containing phospho-ser214, but not with any of the other tau peptides used. PG-5 was shown to react with only the peptide containing phospho-ser409, but not with the equivalent nonphosphorylated peptide or any of the other phospho-tau peptides used.

To demonstrate that the creation of the CP-3 and PG-5 epitopes was dependent on PKA, recombinant tau was reacted 


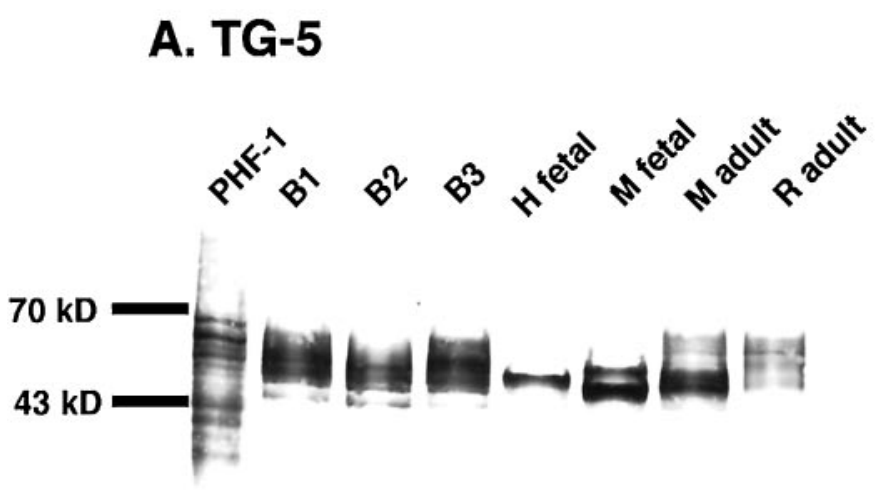

C. CP-13

\section{B. PHF-1}

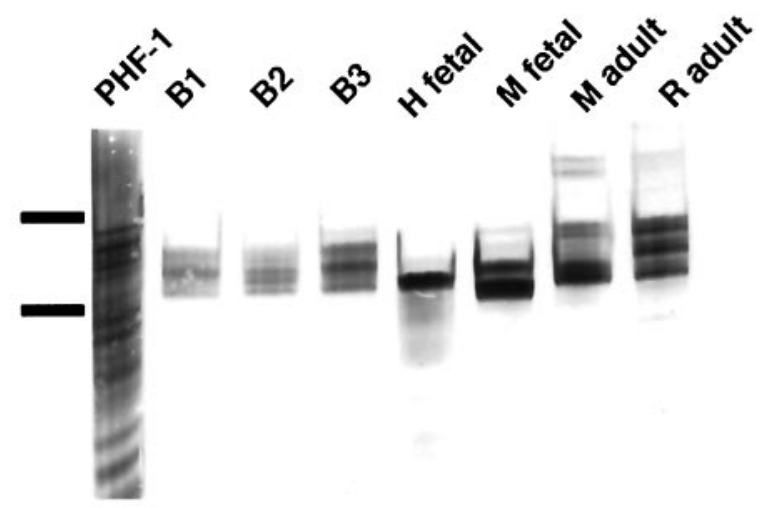

D. CP-9

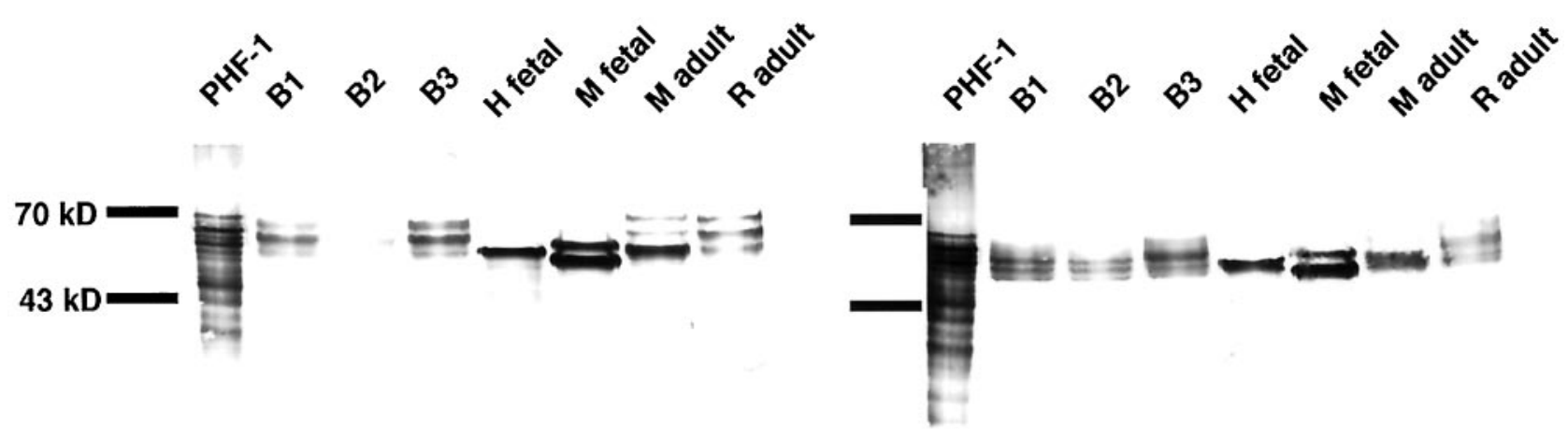

E. CP-3

F. PG-5

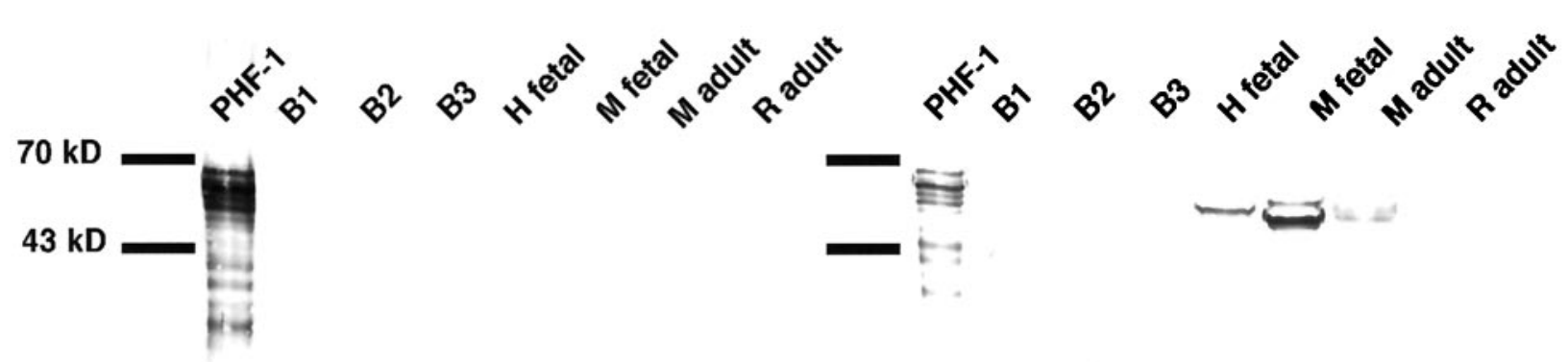

Figure 4. The accumulation of phosphorylations on ser214 and ser409 in tau is a disease-specific event. $A$, A TG-5 Western blot demonstrates total tau levels in each brain preparation from adult human biopsy (B1-B3, three separate cases), human fetal, mouse fetal, mouse adult, and rat adult brain. $B$, A PHF-1 (phospho-ser396/phospho-ser404) Western blot demonstrates abundant phospho-tau in the PHF preparation as well as in the heat-stable brain preparations. $C, \mathrm{CP}-13$ reactivity demonstrates the presence of phospho-ser202 in the same samples used in $A$. $D$, CP-9 reactivity demonstrates the presence of phospho-thr231 in the same samples used in $A . E$, CP-3 exhibits high levels of reactivity toward PHF-tau, but no reactivity with tau in the other samples. F, PG-5 exhibits high levels of reactivity toward PHF-tau as well as some reactivity to human fetal, mouse fetal, and mouse adult brain tau, but does not react with normal human biopsy tissue.

with either PKA, PKC, Cdk5, or GSK-3 $\beta$. Although all kinases were able phosphorylate recombinant tau as determined by autoradiographic analysis, only PKA phosphorylation was shown to create the CP-3 and PG-5 epitopes by Western blot (Fig. 2).

Because previous studies have suggested that both ser214 and ser409 are phosphorylated on AD-tau (Hasegawa et al., 1996), immunostaining was performed on both normal and AD hippocampal tissue sections (Fig. 3). Although CP-3 and PG-5 failed to stain tissues from normal elderly controls, both antibodies were shown to react strongly with neurofibrillary tangles, dystrophic neurites, and neuritic elements of plaques in the AD brain. Additionally, PG-5 was shown to react with structures in the white matter of many AD patients that resembles "coiled bodies" or oligodendrocytic tau inclusions more commonly associated with progressive supranuclear palsy (Fig. $3 H$ ).

To determine if the accumulation of PKA-dependent phospho- 

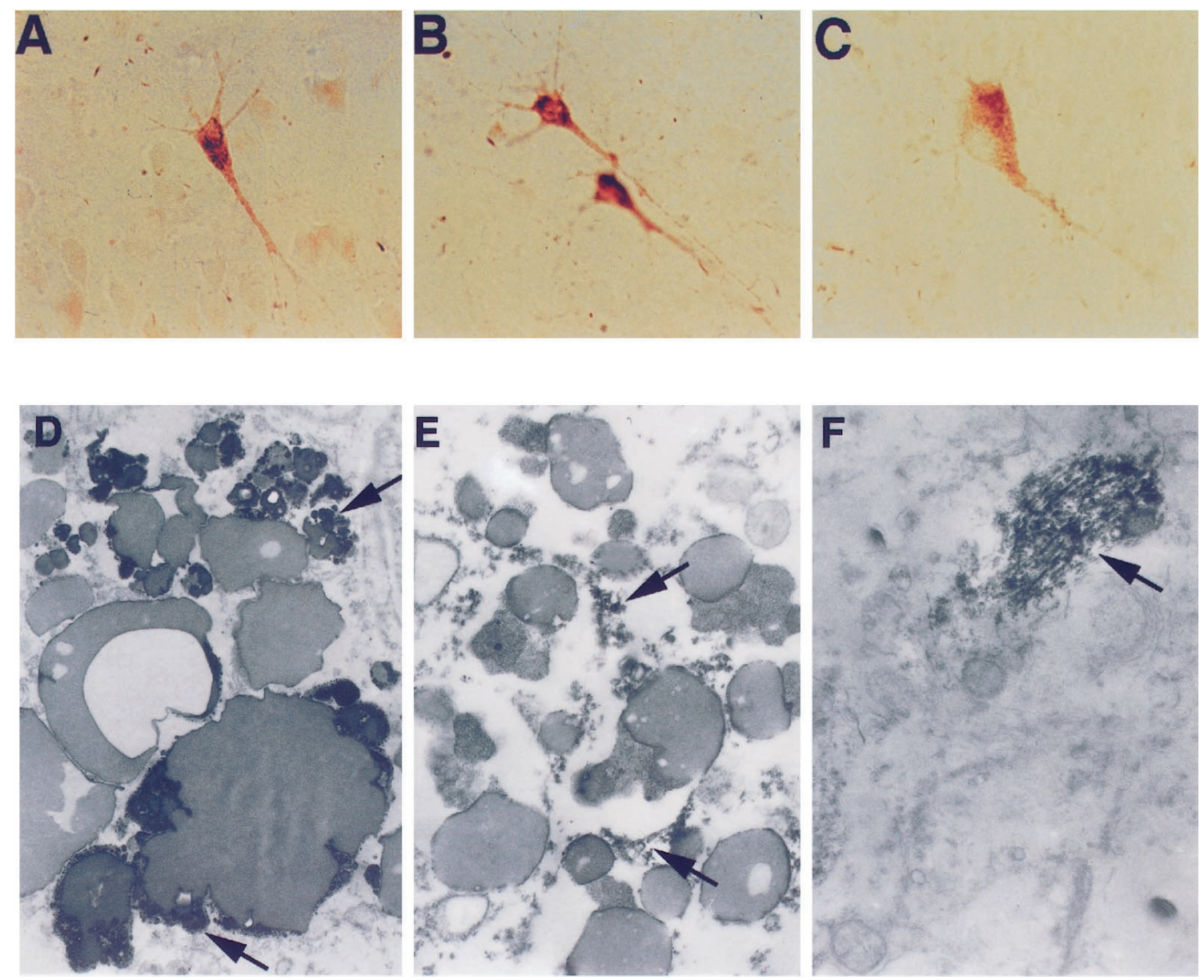

Figure 5. PKA-dependent phosphorylations on tau precede or are coincident with the initial stages of PHF formation and the spread of neurofibrillary pathology in affected neurons in early $\mathrm{AD}$ cases (Braak stage 2). $A-C$, Light microscopy. $A, B, 20 \times$ view of CA2 demonstrating PG-5 reactivity in morphologically "normal" neurons. $C, 40 \times$ view of a PG-5-reactive neuron in CA2 of the hippocampus. Note the punctate perinuclear intensification of immunoreactivity similar to that seen by EM in $D$ and $E$. $D-F$, Electron microscopy. $D$, High-power view of a neuron before PHF formation demonstrating the accumulation of PG-5-reactive material along the periphery of large electron-dense vesicles (arrows). E, PG-5-reactive material can be found in both filamentous and nonfilamentous forms in the vicinity of large electron-dense vesicles in a neuron exhibiting the earliest stages of PHF formation (arrows). F, PG-5 strongly labels early tangles and aggregates of PHF (arrow).

rylations on tau was disease-specific or merely an artifact of the postmortem interval as has been shown for PHF-1 reactivity, CP-3 and PG-5 reactivity was assayed by Western blot against PHF, adult rat, adult mouse, fetal mouse, fetal human, and normal adult human brain heat-stable preparations. As has been shown previously (Matsuo et al., 1994), antibodies recognizing the phosphorylations of ser396/ser404 (PHF-1), ser202 (CP-13), and thr231 (CP-9) exhibit high levels of reactivity against rat, mouse, fetal, and adult human biopsy brain tau (Fig. $4 B-D$ ). In contrast, no CP-3 reactivity is evident except with PHF-tau (Fig. $4 E$ ). PG-5 also reacts well with PHF-tau and exhibits only minimal reactivity that is limited to staining of faint bands in tau preparations from fetal mouse, fetal human, and adult mouse brain (Fig. $4 F$ ). Total tau in these samples is demonstrated by a TG-5 Western blot (Fig. 4A). These data demonstrate that unlike the phospho-tauepitopes recognized by PHF-1 and AT-8, CP-3 and PG-5 reactivity is almost exclusively dependent on an Alzheimer-like disease state in which PKA activity is directed toward the tau molecule.

To determine if the accumulation of PKA-dependent phosphoepitopes on tau contribute to or are a result of the aggregation of tau into PHF in AD, a series of immunostained vibratome brain sections from early AD cases (Braak stage 2; Braak and Braak, 1991) were either analyzed at the light microscopic level or were microdissected and processed for electron microscopy. Analysis of the CA2 region at the light level demonstrates that $\mathrm{PG}-5$ reacts strongly with isolated neurons that appear both morphologically normal and devoid of overt neurofibrillary tangle formation. Although EM analysis demonstrates that the majority of PG-5 reactivity colocalizes with filamentous aggregates and the surrounding area in the soma of neurons, neurons with few or no filamentous inclusions were also detected (Fig. 5). EM analysis of these neurons demonstrates that the electron-dense DAB precipitate localizes to the periphery of large vesicular bodies in which the initial formation of PHF can be seen. These data demonstrate that the accumulation of PKA-dependent phosphorylations on tau can precede overt PHF formation (Fig. 5D), are accumulated in the immediate vicinity of early PHF aggregates (Fig. 5E), and colocalize with the spread of neurofibrillary pathology throughout the afflicted neuron (Fig. $5 F$ ).

Because the discrete localization of PKA-phosphorylated tau by EM analysis suggested that PKA activity was directed toward tau in the early stages of PHF formation, it was important to determine if the PKA holoenzyme complex was also specifically 

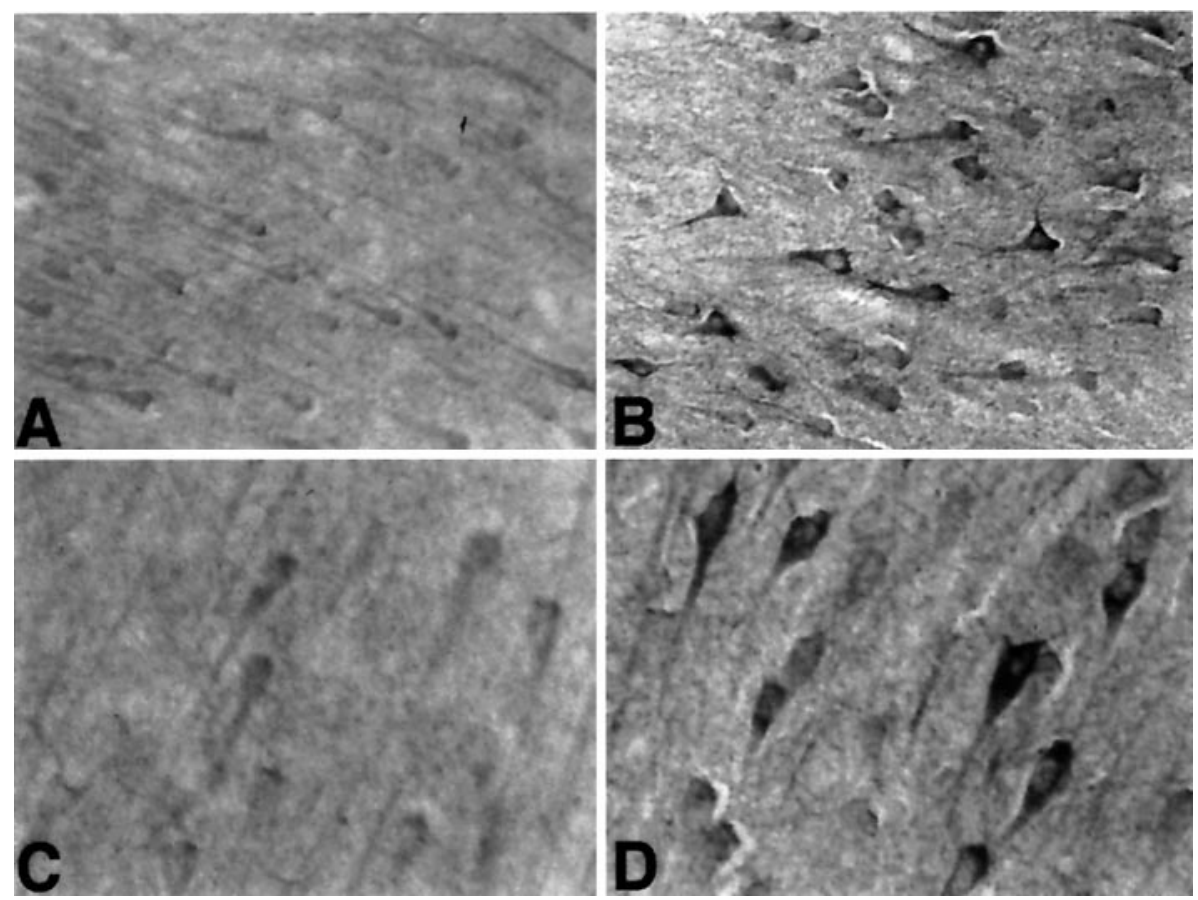

Figure 6. Immunohistochemical localization of AKAP79 in the hippocampus of $\mathrm{AD}$ and normal brain. $A$, Low-power $(10 \times)$ view of normal control brain tissue stained for AKAP 79. Immunoreaction product is localized to the soma and dendritic arbor at low levels. B, Low-power $(10 \times)$ view of AD brain tissue stained for AKAP 79. Neurons that appear to be undergoing the initial stages of neurofibrillary degeneration exhibit enhanced cell body staining with the AKAP 79 monoclonal antibody. $C$, High-power view of $A$. $D$, High-power view of $B$. targeted to the sites of PHF formation. To address this issue, a variety of antibodies recognizing constituents of the PKA holoenzyme complex and several different AKAPs were employed. Although many of these antibodies demonstrated no diseasespecific alterations in staining patterns (data not shown), one monoclonal antibody recognizing AKAP79 strongly labeled the somatodendritic compartment of neurons in areas vulnerable to the progression of neurofibrillary pathology in AD with only weak labeling of neurons in similar brain regions in normal controls (Fig. 6). Additionally, AKAP79 reactivity appeared to colocalize with neurofibrillary pathology in the hippocampus (CA1) recognized by $\mathrm{CP}-3$ and $\mathrm{PG}-5$ in $\mathrm{AD}$ brain tissue by immunocytochemical analysis (Fig. 7). Because of the strong colocalization of AKAP79 immunoreactivity with neurofibrillary pathology in $\mathrm{AD}$, Western blot analysis of five separate immunoaffinity-purified PHF preparations from AD brain homogenates was performed. Figure 8 shows that antibodies to PKA-C $\beta$, PKA-RII $\beta$, and AKAP79, but not calcineurin (an AKAP79-associated protein phosphatase; Coghlan et al., 1995), specifically recognize bands in each of the PHF preparations tested. These data suggest that the accumulation of PKAdependent phosphorylations on tau in AD are a result of directed PKA activity and not merely a result of widespread neuronal PKA activation.

\section{DISCUSSION}

Although it is widely accepted that hyperphosphorylated tau is the major proteinaceous constituent of the neurofibrillary pathology seen in AD brain, there is a great deal of debate over which, if any, of these phosphorylations are disease-specific (Matsuo et al., 1994) and which neuronal kinase systems are involved in the post-translational modification of tau (for review, see Trojanowski and Lee, 1994). Many studies have shown that a variety of neuronal protein kinases are capable of phosphorylating the tau molecule in vitro (for review, see Trojanowski and Lee, 1994). Several of these studies have also demonstrated colocalization of individual protein kinases with the neurofibrillary pathology seen in AD (Hanger et al., 1992; Baumann et al., 1993; Trojanowski et al., 1993; Wood et al., 1993; Arendt et al., 1995). These data suggest that almost any known neuronal protein kinase system could play a role in the pathological hyperphosphorylation of tau in AD.

Recent evidence, however, has demonstrated that most of the so-called "pathological" phosphorylations on tau are found in freshly frozen adult biopsy tau, and that their "accumulation" in AD brain is an artifact of the postmortem actions of neuronal phosphatases in the normal brain (Matsuo et al., 1994; Hasegawa et al., 1996). These findings have spurred the search for true AD-specific alterations in tau that could contribute to the development of the neurofibrillary pathology characteristic of the disease state. Since these discoveries, several groups have reported on the creation and characterization of true AD-specific antibodies, which fall into three classes: (1) those recognizing conformational alterations in AD-tau (Jicha et al., 1997a,b); (2) those requiring multiple phosphorylations in neighboring sites (Hoffmann et al., 1997); and (3) those recognizing the accumulation of true AD-specific phosphorylations that are independent of the postmortem actions of neuronal phosphatases (Matsuo et al., 1994; Hasegawa et al., 1996).

Recent evidence from our laboratory and others has suggested that PKA-dependent phosphorylations on ser214 and ser409 in tau may be an early step in the conversion of normal tau into an AD-like state (Scott et al., 1993; Brandt et al., 1994; Leger et al., 1997; Illenberger et al., 1998; Jicha et al., 1999). To monitor these potentially pathogenic phosphorylations, two new monoclonal antibodies, recognizing phospho-ser214 (CP-3) and phosphoser409 (PG-5), were created. The phosphorylations of ser214 and ser409 on tau are shown to be exclusively created by PKA in vitro, and their accumulation on brain-derived tau is truly a diseasespecific event that precedes and or is coincident with both the initial stages of PHF formation and the spread of neurofibrillary pathology throughout affected neurons in AD. Although previous studies using phosphopeptide mapping strategies have reported 

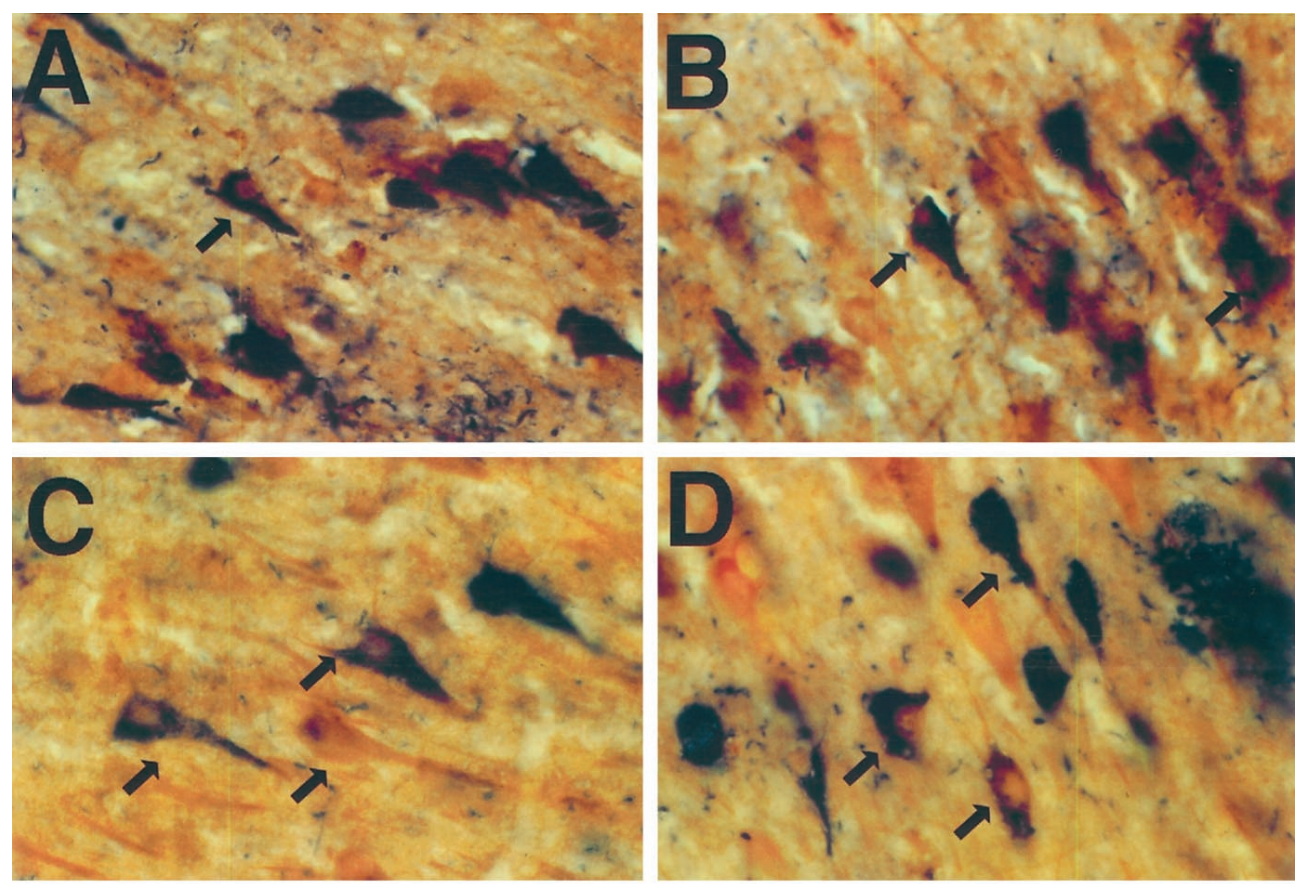

Figure 7. Double immunocytochemistry in CA1 of the hippocampus with CP-3 (A, $C$, and $E$; NBT/BCIP in blue). $A$, AKAP79; C, PKA-C $\beta$; E, PKA-RII (DAB in brown). PG-5 staining $(B, D$, and $F$; NBT/BCIP in blue); $B$, AKAP79; $D$, PKA-C $\beta ; F$, PKA-RII $\beta$ (DAB in brown). Note the colocalization of $\mathrm{CP}-3$ and PG-5 with AKAP79-, PKA-RII $\beta$-, and PKA-C $\beta$-reactive neurons.
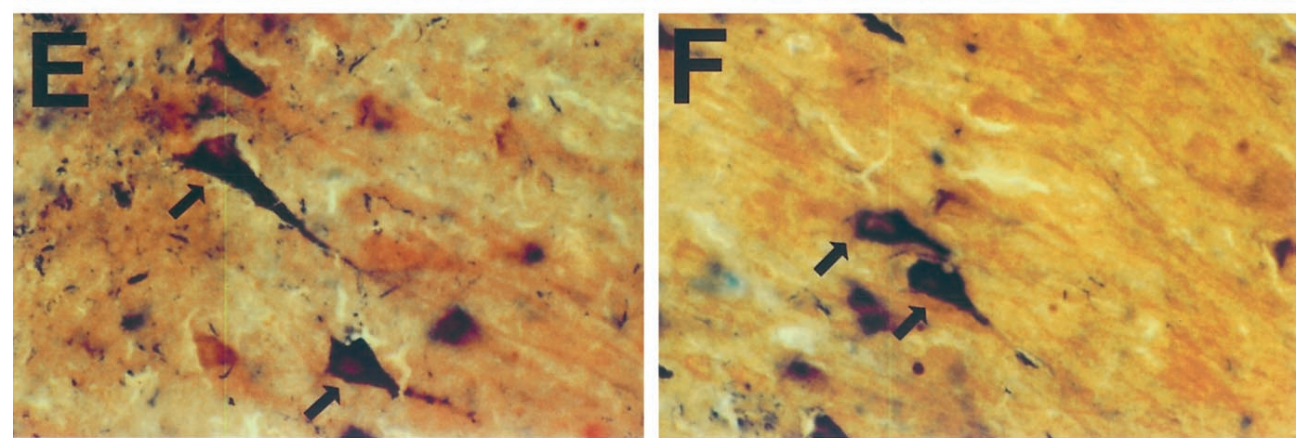

that ser214 and ser409 are phosphorylated in both normal adult and fetal tau (Hasegawa et al., 1992; Yoshida and Ihara, 1993; Hanger et al., 1998), these studies have relied on absolute detection of phosphorylated residues in tau and have not determined the relative levels or stoichiometry of these phosphorylations. The present study, using specific monoclonal antibody detection, argues that the levels of phosphate incorporation into these specific sites is very much higher in PHF-tau than in the normal brain. This has previously been shown to be the case for the AD-specific phosphorylation of ser422 in tau by MAP kinase (Hasegawa et al., 1996). Although phospho-ser422 has been identified in normal adult and fetal tau by phosphopeptide mapping strategies (Hasegawa et al., 1992; Yoshida and Ihara, 1993; Hanger et al., 1998), the development of mAb422 has demonstrated that the accumulation of this phosphorylation on tau is indeed a disease-specific event (Hasegawa et al., 1996).

A more recent report has suggested that antibodies recognizing epitopes dependent on the accumulation of multiple neighboring phosphorylations on tau are disease-specific (Hoffmann et al., 1997). Although we agree that accumulations of certain phosphorylations are indicative of the disease state, we note that the AT100 monoclonal antibody used in this previous study is dependent on the phosphorylations of both thr212 and ser214 in tau (Hoffmann et al., 1997), the latter being the phosphorylation recognized by $\mathrm{CP}-3$ in this study. It is possible that the demonstrated specificity of AT100 for the disease state lies solely in its requirement for ser214 phosphorylation and not in its absolute requirement for dual phosphorylation. Nonetheless, it is apparent that the accumulation of phosphorylations specific for the disease state are clues to which signaling cascades might be altered in AD. The present data suggest that the directed activity of PKA toward

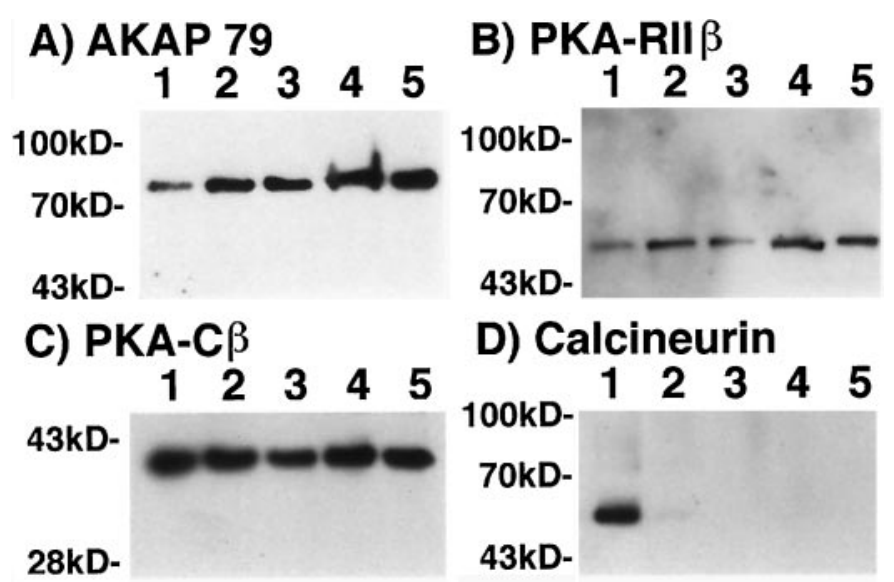

Figure 8. AKAP79, PKA-RII $\beta$, and PKA-C $\beta$ but not calcineurin are tightly associated with PHF preparations from four different severe (Braak stage 5) AD cases (lanes 2-5). Lane 1 is a normal adult whole brain homogenate that serves as a positive control for each of the antibodies used. 
tau is part of the disruption of signal transduction pathways which lead to the development of neurofibrillary pathology in AD, and that both CP-3 and PG-5 may prove to be valuable tools in the early diagnosis of $\mathrm{AD}$-specific alterations in tau.

It is known that PKA activity is controlled by both intracellular levels of cAMP and by the discrete subcellular localization of the holoenzyme complex (for review, see Coghlan et al., 1993; Walsh and Van Patten, 1994). Two major forms of the PKA holoenzyme complex, $\mathrm{C} \beta$-RII $\alpha$ and $\mathrm{C} \beta$-RII $\beta$, are found in the brain regions vulnerable to AD pathology (Cadd and McKnight, 1989; Ludvig et al., 1990; Licameli et al., 1992). The main difference between these complexes is the isoform of the regulatory subunits, RII $\alpha$ and $\operatorname{RII} \beta$, which is the primary determinant of which intracellular proteins (AKAPs) will tether the holoenzyme complex (for review, see Coghlan et al., 1993). RII $\alpha$ has a high affinity for MAP2 (Rubino et al., 1989; Luo et al., 1990), a microtubule-associated protein normally found in the somatodendritic compartment of neurons in which neurofibrillary pathology is found in AD, and RII $\beta$, which has a high affinity for AKAP79 (Coghlan et al., 1995), a member of the AKAP family that localizes to postsynaptic densities in brain regions vulnerable to AD pathology. Antibodies to PKA-C $\beta$, PKA-RII $\beta$, and AKAP79 showed strong associations with neurofibrillary pathology in AD (Figs. 6, 7), whereas RII $\alpha$ staining exhibited a much weaker association (data not shown). These findings suggest that there is a dysregulation of subcellular localization for PKA-C $\beta$, PKA-RII $\beta$, and AKAP79 in $\mathrm{AD}$ which may allow for the specific targeting of tau by activated PKA after elevations in cAMP levels. A sequelae to these alterations in subcellular localization of the PKA holoenzyme complex in AD is that phosphorylation events downstream of PKA activation, such as those of CREB (a transcription factor involved in learning and memory processes), as well as kainate, glutamate, and $\beta$-adrenergic receptors, may not occur in a normal fashion and these signal cascades therefore may fail to function appropriately.

The identification of AKAP79 as a PHF-associated protein, as demonstrated in this study, is interesting in several aspects. First, it should be noted that AKAP79 has previously been shown to be expressed at high levels in cortical regions and hippocampal subfields that are vulnerable to the development of neurofibrillary pathology in AD (Carr et al., 1992; Coghlan et al., 1995; Klauck et al., 1996). It is possible that the presence of AKAP79 in subpopulations of neurons in these regions may be one determinant of which cells develop neurofibrillary pathology. Secondly, it is of interest that in addition to its role in PKA compartmentalization, AKAP79 has been shown to bind and localize calcineurin, a protein phosphatase that has been proposed to exhibit reduced activity in AD (Coghlan et al., 1995; Klauck et al., 1996). The subcellular association of both kinase and phosphatase activities is proposed to allow precise temporal and spatial control of phosphorylation states in key substrates involved in signaling events. Disrupting the association of either PKA or calcineurin with AKAP79 would most likely result in abnormal phosphorylation patterns that would interfere with normal cellular signaling cascades. Although the present study clearly shows an association between PHF, AKAP79, and the PKA-C $\beta$-RII $\beta$ holoenzyme complex, we failed to detect an association between PHF and calcineurin, although double labeling of AD brain tissue clearly demonstrates that both coexist in the same neuronal populations (Billingsley et al., 1995; data not shown). These observations suggest that the proposed inactivity of calcineurin toward PHF in $\mathrm{AD}$ is most likely a result of altered subcellular localization, rather than a direct inactivation of this neuronal phosphatase, as has been suggested previously (for review, see Trojanowski and Lee, 1995). Thus we propose that the PKA-dependent hyperphosphorylation of tau seen in $\mathrm{AD}$ is a result of inappropriate targeting of both neuronal kinases and phosphatases.

It may not be coincidental that PKA has been implicated in the functioning of many of the normal cellular mechanisms that have been postulated to have gone awry in AD. These include the development of long-term potentiation via CREB phosphorylation as well as the regulation of excitotoxic neurotransmission and calcium homeostasis via modulation of NMDA receptors. Although this study and much previous research has suggested that PKA may be involved in the neurofibrillary degeneration seen in $\mathrm{AD}$, it is only now becoming clear how or why such a ubiquitous enzyme could participate in the development of neurofibrillary pathology in specific neuronal subpopulations. Although much research remains to be done, the CP-3 and PG-5 monoclonal antibodies may prove to be valuable tools in the identification of AD-specific alterations in cAMP-mediated signal transduction pathways.

\section{REFERENCES}

Arendt T, Holzer M, Grobmann A, Zedlick D, Bruckner MK (1995) Increased expression and subcellular translocation of the mitogen activated protein kinase and mitogen-activated protein kinase in Alzheimer's disease. Neuroscience 68:5-18.

Baumann K, Mandelkow E-M, Biernat J, Piwnica-Worms H, Mandelkow E (1993) Abnormal Alzheimer'-like phosphorylation of tau-protein by cyclin- dependent kinases cdk2 and cdk5. FEBS Lett. 336:417-424.

Biernat J, Gustke N, Drewes G, Mandelkow E-M, Mandelkow E (1993) Phosphorylation of ser262 strongly reduces binding of tau to microtubules: distinction between PHF-like immunoreactivity and microtubule binding. Neuron 11:153-163.

Billingsley ML, Ellis C, Kincaid RL, Martin J, Schmidt ML, Lee VM-Y, Trojanowski JQ (1995) Calcineurin immunoreactivity in Alzheimer's disease. Exp Neurol 126:178-184.

Braak H, Braak E (1991) Neuropathological staging of Alzheimerrelated changes. Acta Neuropathologica 82:239-259.

Brandt R, Lee G, Teplow DB, Shalloway D, Abdel-Ghany M (1994) Differential effect of phosphorylation and substrate modulation on tau's ability to promote microtubule growth and nucleation. J Biol Chem 269:11776-11782.

Cadd G, McKnight GS (1989) Distinct patterns of cAMP-dependent protein kinase gene expression in mouse brain. Neuron 3:71-79.

Carr DW, Stofko-Hahn RE, Fraser IDC, Cone RD, Scott JD (1992) Localization of the cAMP-dependent protein kinase to the postsynaptic densities by A kinase anchoring proteins. Characterization of AKAP79. J Biol Chem 267:16816-16823.

Coghlan VM, Bergeson SE, Langeberg L, Nilaver G, Scott JD (1993) A-Kinase anchoring proteins: a key to selective activation of cAMPresponsive elements. Mol Cell Biochem 127/128:309-319.

Coghlan VM, Perrino BA, Howard M, Langeberg LK, Hicks JB, Gallitin M, Scott JD (1995) Association of protein kinase A and protein phosphatase 2B with a common anchoring protein. Science 267:108-111.

de St. Groth F, Scheideggers D (1980) Production of monoclonal antibodies: strategy and tactics. J Immunol Methods 35:1-21.

Dreschel DN, Hyman BA, Cobb MH, Kirschner MW (1992) Modulation of the dynamic instability of tubulin assembly by the microtubuleassociated protein tau. Mol Biol Cell 3:1141-1154.

Goedert M, Jakes R (1990) Expression of separate isoforms of human tau protein: correlation with the tau pattern in brain and effects on tubulin polymerization. EMBO J 9:4225-4230.

Goedert M, Spillantini MG, Jakes R, Rutherford D, Crowther RA (1989) Multiple isoforms of human microtubule-associated proteintau: Sequences and localization in neurofibrillary tangles of Alzheimer's- disease. Neuron 3:519-526.

Goedert M, Spillantini MG, Cairns NJ, Crowther RA (1992) Tau proteins of Alzheimer paired helical filaments: abnormal phosphorylation of all six brain isoforms. Neuron 8:159-168.

Greenberg SG, Davies P, Schein JD, Binder LI (1992) Hydrofluoric 
acid- treated $\tau$ PHF proteins display the same biochemical properties as normal $\tau_{\mathrm{PHF}}$ J Biol Chem 267:564-569.

Grundke-Iqbal I, Iqbal K, Tung Y-C, Quinlan M, Wisniewski HM, Binder LI (1986) Abnormal phosphorylation of the microtubuleassociated protein tau in Alzheimer cytoskeletal pathology. Proc Natl Acad Sci USA 83:4913-4917.

Hanger DP, Hughes K, Woodgett JR, Brion J-P, Anderton BH (1992) Glycogen synthase kinase-3 induces Alzheimer's disease-like phosphorylation of tau: generation of paired helical filament epitopes and neuronal localization of the kinase. Neurosci Lett 147:58-62.

Hanger DP, Betts JC, Loviny TLF, Blackstock W P, Anderton BH (1998) New phosphorylation sites identified in hyperphosphorylated tau (paired helical filament-tau) from Alzheimer's disease brain using nanoelectrospray mass spectrometry. J Neurochem 71:2465-2476.

Hasegawa M, Morishima-Kawashima M, Takio K, Suzuki M, Titani K, Ihara Y (1992) Protein sequence and mass spectrometric analysis of tau in the Alzheimer's disease brain. J Biol Chem 267:17047-17054.

Hasegawa M, Jakes R, Crowther RA, Lee VM-Y, Ihara Y, Goedert M (1996) Characterization of mAb AP422, a novel phosphorylationdependent monoclonal antibody against tau protein. FEBS Lett 384:25-30.

Hoffmann R, Lee VM-Y, Leight S, Varga I, Otvos Jr L (1997) Unique Alzheimer's disease paired helical filament specific epitopes involve double phosphorylation at specific sites. Biochemistry 36:8114-8124.

Illenberger S, Zheng-Fischhofer Q, Preuss U, Stamer K, Baumannn K, Trinczek B, Biernat J, Godemann R, Mandelkow E-M, Mandelkow E (1998) The endogenous and cell cycle-dependent phosphorylation of tau protein in living cells: implications for Alzheimer's disease. Mol Biol Cell 9:1495-1512.

Jicha GA, Bowser R, Kazam IG, Davies P (1997a) Alz-50 and MC-1, a new monoclonal antibody raised to paired helical filaments, recognize conformational epitopes on recombinant tau. J Neurosci Res 48:128-132.

Jicha GA, Lane E, Vincent I, Otvos Jr L, Hoffmann R, Davies P (1997b) A conformation- and phosphorylation-dependent antibody recognizing the paired helical filaments of Alzheimer's disease. J Neurochem 69:2087-2095.

Jicha GA, O'Donnell A, Weaver, C, Angeletti R, Davies P (1999) Hierarchical phosphorylation of recombinant tau by the paired helical filament associated protein kinase is dependent on cAMP-dependent protein kinase. J Neurochem 72:214-224.

Kanemaru K, Takio K, Mirua R, Titani K, Ihara Y (1992) Fetal-type phosphorylation of the tau in paired helical filaments. J Neurochem 1667-1675.

Klauck TM, Faux MC, Labudda K, Langeberg LK, Jaken S, Scott JD (1996) Coordination of three signaling enzymes by AKAP79, a mammalian scaffold protein. Science 271:1589-1592.

Lee G (1993) Non-motor microtubule-associated proteins. Curr Opin Cell Biol 5:88-94.

Lee VM, Balin BJ, Otvos Jr L, Trojanowski JQ (1991) A68: a major subunit of the paired helical filaments and derivatized forms of normal tau. Science 251:675-678.

Leger J, Kemp M, Lee G, Brandt R (1997) Conversion of serine to aspartate imitates phosphorylation induced changes in the structure and function of microtubule-associated protein tau. J Biol Chem 272:8441-8446.
Licameli V, Mattiace LA, Erlichman J, Davies P, Dickson D, ShafitZagardo B (1992) Regional localization of the regulatory subunit $(\mathrm{RII} \beta)$ of the type II cAMP-dependent protein kinase in human brain. Brain Res 578:61-68.

Ludvig N, Ribak CE, Scott JD, Rubin CS (1990) Immunocytochemical localization of the neural-specific regulatory subunit of the type II cyclic AMP-dependent protein kinase to post synaptic structures in the rat brain. Brain Res 520:90-102.

Luo Z, Shafit-Zagardo B, Erlichman J (1990) Identification of the MAP2- and p75-binding domain in the regulatory subunit (RII beta) of type II cAMP-dependent protein kinase. Cloning and expression of the cDNA for bovine brain RII beta. J Biol Chem 265:21804-21810.

Matsuo ES, Shin R-W, Billingsley ML, Van deVorde A, O'Connor M, Trojanowski JQ, Lee VM-Y (1994) Biopsy-derived adult human tau is phosphorylated at many of the same sites as Alzheimer's disease paired helical filament tau. Neuron 13:989-1002.

Otvos Jr L, Feiner L, Lang E, Szendrei GI, Goedert M, Lee VM-Y (1994) Monoclonal antibody PHF-1 recognizes tau protein phosphorylated at serine residues 396 and 404. J Neurosci Res 39:669-673.

Paudel HK, Lew J, Ali Z, Wang JH (1993) Brain proline-directed kinase phosphorylates tau on sites that are abnormally phosphorylated in tau associated with Alzheimer's paired helical filaments. J Biol Chem 268:23512-23518.

Raffaelli N, Yamauchi PS, Purich DL (1992) Microtubule-associated protein autophosphorylation alters in vitro microtubule dynamic instability. FEBS Lett 296:21-24.

Rubino HM, Dammerman M, Shafit-Zagardo B, Erlichman J (1989) Localization and characterization of the binding site for the regulatory subunit of type II cAMP-dependent protein kinase on MAP2. Neuron 3:631-638.

Scott CW, Spreen RC, Herman JL, Chow FP, Davison MD, Young J, Caputo CB (1993) Phosphorylation of recombinant tau by cAMPdependent protein kinase. J Biol Chem 268:1166-1173.

Trojanowski JQ, Lee VM-Y (1994) Paired helical filament $\tau$ in Alzheimer's disease the kinase connection. Am J Pathol 144:449-453.

Trojanowski JQ, Lee VM-Y (1995) Phosphorylation of paired helical filament tau in Alzheimer's disease neurofibrillary lesions: focusing on phosphatases. FASEB J 9:1570-1576.

Trojanowski JQ, Mawal-Dewan M, Schmidt ML, Martin J, Lee VM-Y (1993) Localization of the mitogen activated protein kinase ERK2 in Alzheimer's disease neurofibrillary tangles and senile plaque neurites. Brain Res 618:333-337.

Vincent IJ, Rosado M, Kim E, Davies P (1994) Increased production of paired helical filament epitopes in a cell culture system reduces the turnover of t. J Neurochem 62:715-723.

Walsh DA, Van Patten SM (1994) Multiple pathway signal transduction by the cAMP-dependent protein kinase. FASEB J 8:1227-1236.

Wood JG, Mirra S, Pollock NJ, Binder LI (1986) Neurofibrillary tangles of Alzheimer's disease share antigenic determinants with the axonal microtubule-associated protein tau. Proc Natl Acad Sci USA 83:4040-4043.

Wood JG, Lu Q, Reich C, Zinsmeister P (1993) Proline-directed kinase systems in Alzheimer's disease pathology. Neurosci Lett 156:83-86.

Yoshida H, Ihara Y (1993) Tau in paired helical filaments is functionally distinct from fetal tau: assembly incompetence of paired helical filament tau. J Neurochem 61:1183-1186. 